University of Nebraska - Lincoln

DigitalCommons@University of Nebraska - Lincoln

April 1984

\title{
A SEARCH FOR CHROMOSPHERIC EMISSION IN TU CASSIOPEIAE SPECTRA
}

Arne A. Henden

Department of Astronomy, Indiana University, Bloomington, Indiana

Robert H. Cornett

Systems and Applied Sciences Corporation, Hyattsville, Maryland

Edward G. Schmidt

University of Nebraska-Lincoln, eschmidt1@unl.edu

Follow this and additional works at: https://digitalcommons.unl.edu/physicsschmidt

Part of the Physics Commons

Henden, Arne A.; Cornett, Robert H.; and Schmidt, Edward G., "A SEARCH FOR CHROMOSPHERIC EMISSION IN TU CASSIOPEIAE SPECTRA" (1984). Edward Schmidt Publications. 30.

https://digitalcommons.unl.edu/physicsschmidt/30

This Article is brought to you for free and open access by the Research Papers in Physics and Astronomy at DigitalCommons@University of Nebraska - Lincoln. It has been accepted for inclusion in Edward Schmidt Publications by an authorized administrator of DigitalCommons@University of Nebraska - Lincoln. 


\title{
A SEARCH FOR CHROMOSPHERIC EMISSION IN TU CASSIOPEIAE SPECTRA
}

\author{
ARNE A. HENDEN ${ }^{\circ}$ \\ Department of Astronomy, Indiana University, Bloomington, Indiana 47405 \\ ROBERT H. CORNETT \\ Systems and Applied Sciences Corporation, 5809 Annapolis Road, Suite 200, Hyattsville, Maryland 20784 \\ AND \\ EDWARD G. SCHMIDT \\ Department of Physics and Astronomy, University of Nebraska, Lincoln, Nebraska 68588 \\ Received 1983 November 12, revised 1984 January 26
}

\begin{abstract}
We have searched high-resolution spectra of the multimode cepheid TU Cas for evidence of chromospheric emission. Spectral regions covered include the Ca II near-IR triplet near $8600 \AA, \mathrm{H} \alpha$, Ca II H and K, Mg II h and k; phase coverage in both primary and secondary modes was reasonably complete. There was no evidence for changes other than those caused by temperature variation. The observed lack of emission makes unlikely current mass loss (and resulting helium enrichment) by means of stellar winds.
\end{abstract}

Key words: cepheids-spectra-TU Cas

\section{Introduction}

Multimode cepheids are interesting from a theoretical viewpoint because observational masses, computed from period ratios, are three to four times larger than those derived from no-mass-loss evolutionary calculations (Iben 1974). This discrepancy has led to reexamination of evolutionary and pulsational models; the most promising possible resolution of the problem (Cox, Hodson, and King 1979) requires helium-rich convective zones, mixed downward by an inverted $\mu$-gradient instability. Helium enrichment could be accomplished in multimode cepheids by a stellar wind, which could be observed as emission in the cores of several chromospheric absorption lines. Barrell (1978) observed $\mathrm{H} \alpha$ emission in multimode cepheids.

We have observed TU Cassiopeiae, the brightest multimode cepheid, at high spectral resolutions at wavelengths surrounding the $\mathrm{Ca}$ II near-infrared triplet at $8600 \AA, H \alpha, C a$ II $\mathrm{H}$ and $\mathrm{K}$, and $\mathrm{Mg}$ II $\mathrm{h}$ and $\mathrm{k}$. Several sets of these observations were made simultaneously, at times that gave good phase coverage. We report here the results of those observations.

\section{Observations}

\section{A. Near-IR Spectra}

The near-IR spectra were taken in 1980 with the 1728-channel Reticon on the coude spectrograph of the McDonald 2.1-m telescope. A $250 \AA$ wide region was covered, centered on the $\mathrm{Ca}$ II near-infrared triplet at

"Now at Systems and Applied Sciences Corp., 5809 Annapolis Road, Hyattsville, MD 20784.
$8498 \AA, 8542 \AA$, and $8662 \AA$, with a resolution of $0.3 \AA$. Eight exposures each of $\mathrm{Tu}$ Cas and a comparison star (TU Vulpeculae) were made. The data were smoothed and spurious data, caused by radiation hits during the 90-minute exposures, were removed. The Ca II triplet and weaker lines were examined for changes with phase and for evidence of possible emission. No evidence for changes other than those caused by temperature variation was observed, and no emission was found in the spectra.

\section{B. Optical Spectra}

The coudé feed telescope at Kitt Peak National Observatory was used to obtain spectrograms of TU Cas and a number of other cepheids between January 1980 and October 1981. During October 1981 the observing runs were simultaneous with the IUE observations of TU Cas. A Carnegie image tube with an S20 cathode was used for these spectra. The resolution on the plate with the grating and camera combination used was $0.4 \AA$. We obtained 29 spectrograms centered on $\mathrm{Ca}^{I I_{\mathrm{II}} \mathrm{H}}$ and $\mathrm{K}$ and, during the runs which were simultaneous with the IUE observations, 15 spectra centered on $\mathrm{H} \alpha$. The spectrograms centered at $\mathrm{Ca}$ II $\mathrm{H}$ and $\mathrm{K}$ were overexposed in the continuum in order to allow a search of the core for emission features. The spectra were digitized with the PDS microdensitometer at Kitt Peak and tracings in intensity units were produced.

The tracings and original plates of the Ca II spectra were examined and compared with those of other cepheids. As expected, several longer-period stars showed easily visible emission features but none were visible in the shorter-period cepheids. In the case of TU Cas there was 


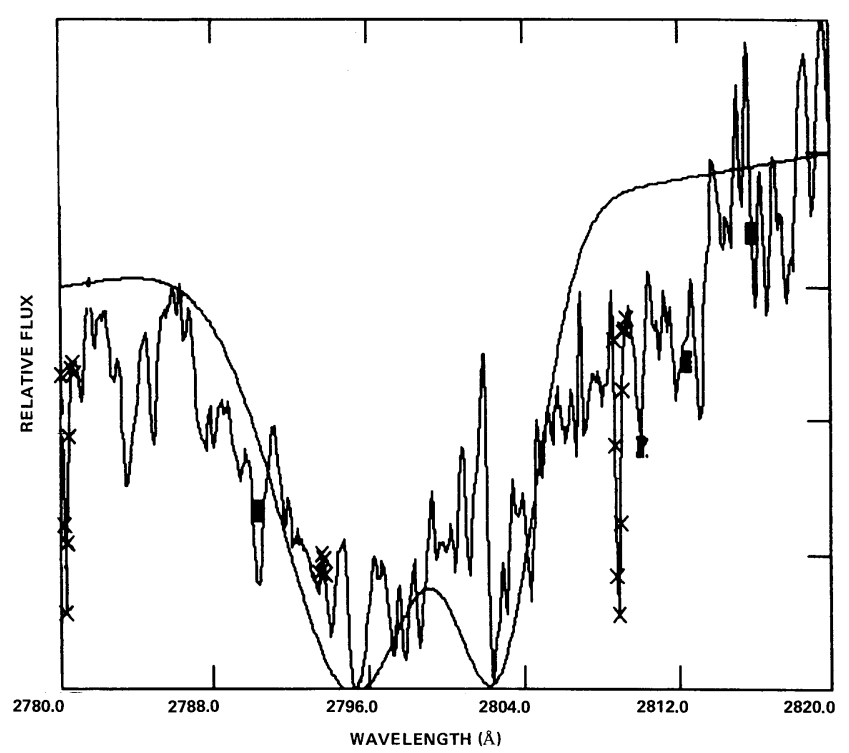

FIG. 1-A portion of a long-wavelength, high-resolution IUE spectrum of TU Cas near the $2800 \AA \mathrm{Mg}$ II doublet. Primary phase was 0.9 , secondary 0.1 .

not a single instance among the 29 spectra in which any emission was visible either in the tracings or on the original spectra. A comparison with spectra of RT Aurigae showed that the profiles in the two stars were very similar.

The profiles of $\mathrm{H} \alpha$ all proved to be symmetrical both in the wings and in the cores. There was no indication of any emission similar to that shown by Barrell (1978) in any of the spectra. Spectra of RT Aur taken during the same run showed similar profiles.

\section{UV Spectra}

IUE was used in October 1981 to obtain six spectra of TU Cas and three of the comparison cepheid RT Aur. Two TU Cas spectra were made with the LWR camera with exposure times of 3.5 and 6.0 hours. The portion of the 6.0-hour exposure surrounding the $\mathrm{Mg}$ II doublet at $2800 \AA$, made at primary phase 0.9 and secondary phase 0.1 , is shown in Figure 1. The smooth solid line is a double Gaussian fit by eye. The spectrum may show weak evidence for emission in the core of the lines. Interpretation is complicated by noise and interstellar absorption.

Low-dispersion IUE spectra of TU Cas made in the same time interval show only temperature-induced changes. Comparison of spectra made using the large (10 $\times 20$ arc sec) and small ( 3 arc sec) entrance apertures available on IUE shows no evidence for a hot companion star. All UV spectra show features strongly reminiscent of $\delta$ Cephei, with no evidence of abnormality.

\section{Discussion}

The detection of emission features in cepheids is made

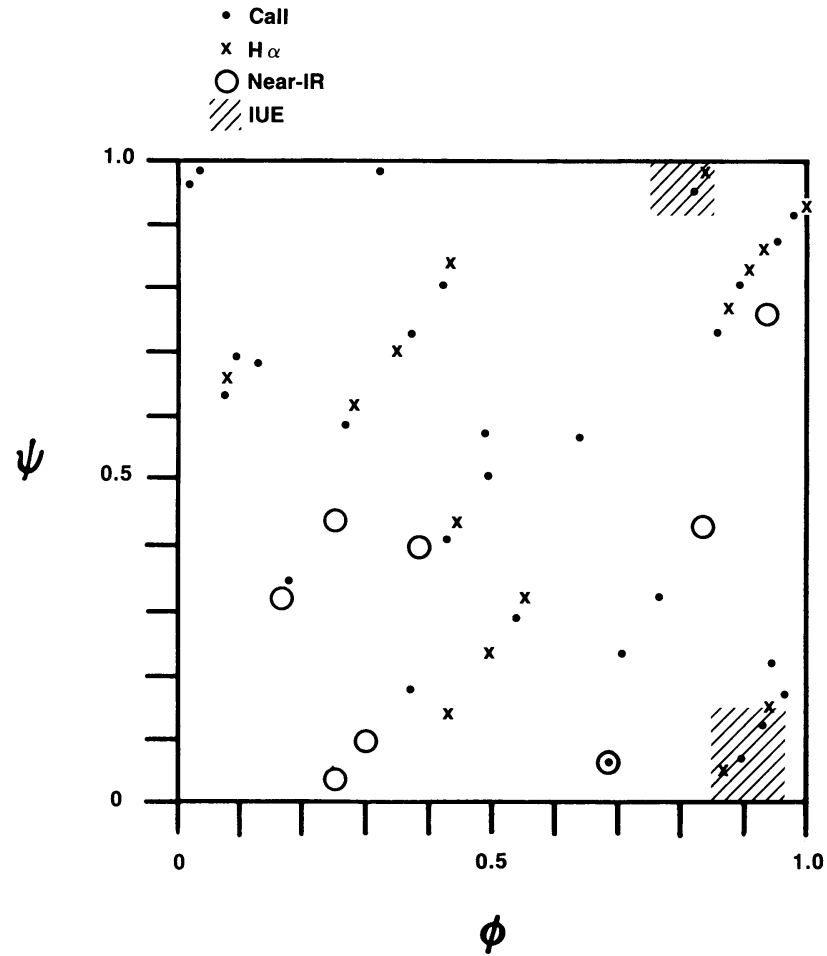

FIG. 2-The $\phi, \psi$ plane coverage of reported TU Cas spectra. $\phi$ is the primary mode phase and $\psi$ is the secondary mode phase. Symbols are described in the text.

difficult by the fact that these features are usually of short duration and are easily missed unless there is good phase coverage. This is further complicated in the case of a multimode cepheid because the phases relative to both periods must be considered. We have used the ephemerides of Faulkner (1977) to calculate phases for the primary period, denoted by $\phi$, and for the secondary period, $\psi$. In Figure 2 we have plotted the phases of all the spectra. Solid circles indicate spectra centered on the $\mathrm{Ca}$ II doublet, crosses indicate spectra centered on $\mathrm{H} \alpha$, open circles mark near-IR spectra, and the shaded regions mark the high-dispersion $I \hat{U E} \mathrm{Mg}$ II spectra. It can be seen that the spectra are well distributed in both phases and our coverage of the $\phi, \psi$ plane is quite good.

Barrell (1978) found evidence for $\mathrm{H} \alpha$ emission of varying strength in seven out of nine multimode cepheids in the primary phase range 0.7 to 1.0 . Our observations of TU Cas include seven $\mathrm{H} \alpha, 13 \mathrm{Ca}$ II, three nearIR Ca IL and two Mg II spectra in that primary phase interval. We conclude that it is unlikely that there is chromospheric emission from TU Cas at any phase, and find no evidence for a stellar wind from that star.

\section{REFERENCES}

Barrell, S. L. 1978, Ap. J. (Letters) 226, L141.

Cox, A. N., Hodson, S. W., and King, D. S. 1979, Ap. J. (Letters) 230, L109.

Faulkner, 1977, Ap. J. 218, 209.

Iben, I., Jr. 1974, Ann. Rev. Astr. Ap. 12, 215. 